\title{
Proposed Search for Small Violations of Bose Statistics in Molecular Spectra $(*)$.
}

\author{
G. M. Tino
}

Dipartimento di Scienze Fisiche, Università di Napoli "Federico II"

Mostra d'Oltremare, Pad. 20, 80125 Napoli, Italia

(ricevuto il 30 Giugno 1994; approvato il 5 Settembre 1994)

Summary. - I discuss the possibility of performing a test of the symmetrization postulate for bosons by high-sensitivity spectroscopy of ${ }^{16} \mathrm{O}_{2}$. I show that the sensitivity required to give significant information can be easily achieved by well-developed spectroscopic methods.

PACS 42.50.Wm - Optical tests of fundamental laws and forces (including QED, Bell inequalities, parity-conservation violation, relativistic effects, gravity waves).

\section{1. - Introduction.}

The symmetrization postulate is a fundamental postulate in quantum mechanics. It states that in a system containing identical particles, the only possible states are either all symmetrical or all antisymmetrical with respect to permutations of the particles. In the first case the particles are called bosons, in the second case they are called fermions.

Different statistical properties result in the two cases from the postulate. Bosons follow Bose-Einstein statistics, fermions obey Fermi-Dirac statistics.

Experiments indicate that elementary particles with half-integer values of spin are fermions, while those with integer spin are bosons.

In fact, quantum mechanics would allow also symmetries different from those imposed by the symmetrization postulate. The possibility of quantum-mechanical systems following statistics different from Bose and Fermi statistics has been proposed in several areas of physics [1-13]. Although experimental evidence allows to exclude gross differences with respect to Bose and Fermi statistics, theories have been developed allowing also for small deviations from conventional statistics, which might have been masked in the experiments performed so far.

(*) The author of this paper has agreed to not receive the proofs for correction. 
Indeed, in spite of the importance of the symmetrization postulate in the understanding of many phenomena in physics, and contrary to what is commonly believed, its validity has not been tested accurately until recently. In fact, only one accurate experiment exists checking the validity of the Pauli principle for electrons [14].

The experiment I discuss here would allow an accurate test of the validity of the symmetrization postulate for bosons by looking for ${ }^{16} \mathrm{O}_{2}$ molecules in states which are forbidden according to the postulate.

I show that significative data can be obtained by using well-developed methods of laser spectroscopy. It is sufficient to look for lines that a molecular spectroscopist would never look for.

\section{2. - Symmetrization postulate and molecular spectra.}

As is well known, the symmetrization postulate allows to understand important features of the spectra of molecules containing identical nuclei and, in particular, the spectra of homonuclear diatomic molecules [15].

According to the Born-Oppenheimer approximation and neglecting the coupling of the nuclear spin with the rest of the molecule (which is not important for the experiment proposed here since the spin of ${ }^{16} \mathrm{O}$ nucleus is zero), the total wave function $\psi_{\text {tn }}$ can be written in the form

$$
\psi_{\mathrm{tn}}=\psi_{\mathrm{e}} \cdot \psi_{\mathrm{v}} \cdot \psi_{\mathrm{r}} \cdot \psi_{\mathrm{n}}=\psi_{\mathrm{t}} \cdot \psi_{\mathrm{n}},
$$

where $\psi_{\mathrm{e}}, \psi_{\mathrm{v}}$ and $\psi_{\mathrm{r}}$ are, respectively, the electronic, vibrational and rotational wave function and $\psi_{\mathrm{n}}$ is the nuclear spin function.

According to the symmetrization postulate, the total wave function must be symmetric or antisymmetric in the exchange of the identical nuclei; $\psi_{\text {tn }}$ will not change if the nuclei are bosons, it will change sign if the nuclei are fermions. Taking into account the symmetry of the electronic, vibrational and nuclear functions, it is possible to explain the relative intensities of lines starting from different rotational levels.

The ${ }^{16} \mathrm{O}_{2}$ molecule represents a particularly simple case. The nuclear spin of ${ }^{16} \mathrm{O}$ is zero and the nuclear spin function is therefore obviously symmetric. The vibrational wave function $\psi_{v}$ is also unaltered in the exchange of the nuclei because it depends only on the magnitude of the internuclear distance. Since the total wave function $\psi_{\text {tn }}$ must be symmetric, only the states corresponding to even (odd) rotational quantum are allowed if $\psi_{\mathrm{e}}$ is symmetric (antisymmetric).

The ground state of the ${ }^{16} \mathrm{O}_{2}$ molecule is a ${ }^{3} \Sigma_{\mathrm{g}}^{-}$state, which is antisymmetric under the exchange of the two nuclei. The rotational states corresponding to even rotational numbers are therefore forbidden according to the postulate. Indeed, only alternate lines have been observed in the spectra [15].

On the other hand, it is clear that a violation of the symmetrization postulate would allow also states corresponding to even rotational numbers. Searching for transitions starting from these levels corresponds then to searching for violations of the symmetrization postulate. This is indeed the idea of the proposed experiment which is discussed in the following. 


\section{3. - Possible violations of Bose and Fermi statistics.}

31. Theoretical background. - It is not the purpose of this paper to discuss the theories which account for statistics different from Bose and Fermi for identical particles. Only some basic concepts are sketched in this section in order to set the idea underlying the proposed experiment in a more general frame.

Before proceeding in the discussion, it is worth emphasizing that on the basis of experimental evidence it is easy to exclude gross differences with respect to Bose and Fermi statistics. It is then of interest to consider those theories which give rise to small deviations from the conventional statistics. Such deviations can be expressed as a different symmetry of the state under particle exchange or, in a Fock-space representation, as a deformation of the algebra of the creation and annihilation operators.

The first attempt to go beyond Bose and Fermi statistics was made in ref.[1] introducing intermediate statistics in which up to $n$ particles can occupy a given quantum state. Fermi statistics is recovered for $n=1$ and Bose statistics corresponds to $n \rightarrow \infty$. This idea led to the developments of a generalized field theory [2] in which the field operators obey trilinear commutation relations of the form

$$
\begin{gathered}
{\left[\left[a_{k}^{\dagger}, a_{l}\right]_{ \pm}, a_{m}\right]_{-}=-2 \delta_{k m} a_{l},} \\
{\left[\left[a_{k}, a_{l}\right]_{ \pm}, a_{m}\right]_{-}=0,}
\end{gathered}
$$

where $\left[a_{i}, a_{j}\right]_{ \pm}=a_{i} a_{j} \pm a_{j} a_{i}$, instead of the usual bilinear commutation and anticommutation relations $\left[a_{i}, a_{j}^{\dagger}\right]_{+}=\delta_{i j}$ and $\left[a_{i}, a_{j}^{\dagger}\right]_{-}=\delta_{i j}$ valid for fermions and bosons, respectively. This theory, called parastatistics, is a consistent local quantum field theory. It predicts, however, gross violations of statistics which, as already mentioned, is in evident contrast with physical experience.

It is of interest then to consider the possibility of statistics which can give rise to small violations of the symmetrization postulate. This means that in the case of electrons, for example, one can write the two-electron density matrix $\rho_{2}$ in the form

$$
\rho_{2}=\left(1-\frac{1}{2} \beta^{2}\right) \rho_{\mathrm{a}}+\frac{1}{2} \beta^{2} \rho_{\mathrm{s}},
$$

where $\rho_{\text {a(s) }}$ is the antisymmetric (symmetric) two-electron density matrix. A pair of electrons will be in the normal antisymmetric state with probability $\left(1-(1 / 2) \beta^{2}\right)$ and in the anomalous symmetric state with probability $(1 / 2) \beta^{2}$. In the literature, (1/2) $\beta^{2}$ is usually defined as the probability that a pair of identical particles is in the anomalous state. In the case of bosons, therefore, $\rho_{\mathrm{a}}$ and $\rho_{\mathrm{s}}$ are exchanged in eq. (3), so that $(1 / 2) \beta^{2}$ gives the probability that the system is in the antisymmetric state.

A theory allowing a continuous interpolation between Bose and Fermi statistics was developed in recent years $[6-8,16]$. The commutation and anticommutation relations of creation and annihilation operators are replaced by generalized bilinear commutation relations depending on a parameter $q$ ( $q$-mutators):

$$
a_{k} a_{l}^{\dagger}-q a_{l}^{\dagger} a_{k}=\delta_{k l}, \quad-1 \leqslant q \leqslant 1,
$$

with the vacuum condition $a_{k}|0\rangle=0$. 
The particles which obey this type of statistics are called «quons». Bose and Fermi statistics are recovered in the cases $q=1$ and $q=-1$, respectively.

It can be shown [8] that, assuming the commutation relation in eq. (4), the two-electron density matrix can be expressed in terms of $q$ as

$$
\rho_{2}=\frac{1}{2}(1-q) \rho_{\mathrm{a}}+\frac{1}{2}(1+q) \rho_{\mathrm{s}} \text {, }
$$

which provides a connection between $q$ and $\beta^{2}$. The importance of quon statistics is that it gives an example of an interpolation between Fermi and Bose statistics. It has been shown[16] that this interpolation preserves positivity of norms and the non-relativistic form of locality. Other aspects are still doubtful as the possibility to allow local observables in a relativistic theory or to account for the existence of antiparticles $[16,17]$.

A different approach to small violations of Fermi and Bose statistics has also been studied using deformations of the trilinear commutation relations introduced in ref. [2]. In these theories, however, negative probabilities appear $[3,5,18]$.

This brief overview, which is necessarily incomplete, shows that symmetries different from those imposed by the symmetrization postulate can in principle be conceived in the frame of quantum mechanics and lead to statistics different from Bose and Fermi statistics. Although the form of these new statistics may appear not as clear as the other ones, it is worth observing that, as stated in ref. [19], we have neither a complete understanding nor an elementary explanation of the spin-statistics connection. In the following subsection, it is shown that we do not even have a clear phenomenological support for the symmetrization postulate.

32. Experiments. - One can envisage three types of experiments to search for deviations from Bose or Fermi statistics [8]: 1) to detect an accumulation of particles in anomalous states, 2) to detect transitions to anomalous states, and 3) to detect deviations from the usual statistical properties of many-particle systems. Type-3) experiments are usually considered as proofs of the validity of the symmetrization postulate. In fact, their accuracy is low and they would hardly allow to show any small deviation. Type-1) and -2) experiments can be much more accurate, provided sensitive enough detection methods are used.

The accuracy of the existing data is very different for Fermi and Bose statistics [20]. In fact, in the case of electrons a high-precision experiment was performed by Ramberg and Snow[14]. They passed a current through a strip of copper and looked for X-ray transitions due to an electron in the $n=2$ level making a transition to the $n=1$ level. Since no such a signal was found, they concluded that the probability that a new electron introduced into copper would form a mixed symmetry state with respect to the electrons already present in the copper sample, thus violating the Pauli principle, is $(1 / 2) \beta^{2} \leqslant 1.7 \cdot 10^{-26}$. In the classification introduced above this is an experiment of type 2 ).

In the case of Bose statistics, no accurate test exists at present. The early measurements by Herzberg on $\mathrm{O}_{2}$ spectra showed that lines involving even $K$ rotational levels of the ground state were missing in the spectra but the accuracy of the data is too low to use them to set a significative bound to possible small violations. Reinterpreted as a search for small violations of Bose statistics, they would lead to an upper limit of $\beta^{2} \leqslant 10^{-4}$. 
As discussed in ref. [20], one can constrain $\beta^{2}$ by assuming that the decay $\mathrm{K}_{\mathrm{L}}^{0} \rightarrow$ $\rightarrow \pi^{+} \pi^{-}$which is usually considered to come from $C P$ violation, is due to violation of Bose statistics. This would give $\beta^{2} \leqslant 10^{-6}$.

A limit $\beta^{2} \leqslant 10^{-6}$ to violations of Bose statistics for photons was inferred in ref. [9] on the basis of the presently attainable laser intensities.

Other tests of Fermi and Bose statistics using atoms [21] or molecules [22] have been proposed but no results have been reported so far.

\section{4. - Proposed experiment.}

4*1. General idea. - The basic idea of the proposed experiment is rather simple. The aim is to distinguish $\mathrm{O}_{2}$ molecules in states with "wrong" symmetry (that is, in states with even values of the rotational number $K$ ) from a large number of molecules in the «right» symmetry states. Therefore, the problem is the same as that of the detection of traces of an element, which is typical in atomic and molecular spectroscopy. Techniques have been demonstrated with sensitivities which can approach the single-particle detection [23].

In this section, I will consider a possible experiment of absorption spectroscopy. The important point is whether the attainable sensitivity is high enough to give significative information. I will show that this is possible even with a relatively simple apparatus.

Recently, we demonstrated the possibility of detecting the magnetic-dipole transitions of the $X^{3} \Sigma_{\mathrm{g}}^{-} \rightarrow b^{1} \Sigma_{\mathrm{g}}^{+}$atmospheric system of $\mathrm{O}_{2}$ at $762 \mathrm{~nm}(0-0$ band) by simple absorption spectroscopy [24]. In spite of the very small absorption coefficient $a \sim 5 \cdot 10^{-7}$ Torr $^{-1} \mathrm{~cm}^{-1}$ of these transitions, spectra were recorded at pressures as low as a few Torrs with an absorption path of one meter thanks to the very low amplitude noise of the semiconductor diode lasers used as tunable radiation source. Transitions of the ${ }^{P} P$ branch [15] starting from levels corresponding to the values $K=$ $=1,3,9$ of the rotational quantum number were investigated. A systematic study was performed of the dependence of the line intensity and broadening on the gas temperature. In particular, the signal from the ${ }^{P} P(K=1)$ line was found to increase by one order of magnitude when the gas temperature is reduced from room temperature to about $100 \mathrm{~K}$.

In the experiment proposed here, one would use essentially the same experimental scheme to try to detect transitions involving anomalous-symmetry states among the allowed transitions. A good candidate is for example the ${ }^{P} P(K=2)$ transition whose expected position is far from other possible interfering lines.

With a proper optimization and calibration of the sensitivity of the apparatus one can expect to achieve an accuracy high enough to improve by several orders of magnitude the bounds to violations of the statistics, as described in the following.

It is worth mentioning that $\mathrm{O}_{2}$ is not the only molecule on which this experiment can be performed. Other diatomic molecules could be investigated such as $\mathrm{He}_{2}, \mathrm{C}_{2}, \mathrm{~S}_{2}$, or $\mathrm{Se}_{2}$. The production of these species, however, in a proper environment for high-sensitivity spectroscopy poses serious problems. Detection of dipole-allowed transitions between anomalous-symmetry states of triatomic molecules such as $\mathrm{C}^{16} \mathrm{O}_{2}$ is also possible [22]. Compared to other molecules, however, $\mathrm{O}_{2}$ offers two advantages: first, it is the simplest system allowing this kind of experiment, which will simplify the interpretation of the results. Second, 
high-sensitivity detection techniques can be easily implemented in the relevant spectral region, as discussed in the following.

42. Experimental. - The apparatus required for the proposed experiment is a typical apparatus for laser absorption spectroscopy. It is made of a) a laser source, $b$ ) an absorption cell, and $c$ ) a detection apparatus. In the following, a possible scheme is briefly described in order to determine what is the accuracy one can expect to achieve.

a) Laser source: in order to investigate transitions at $762 \mathrm{~nm}$, AlGaAs diode lasers emitting at $780 \mathrm{~nm}$ at room temperature can be used. They can be tuned down to about $762 \mathrm{~nm}$ by cooling them at $\sim 240 \mathrm{~K}$ [24].

Frequency stabilization and control can be achieved using an external cavity made with a diffraction grating $[25,26]$. This allows a fine tuning of the emission frequency and the suppression of side modes which would prevent accurate absorption measurements.

The amplitude noise of these laser sources is very low. In fact, it can be reduced close to the shot noise limit using negative electronic feedback to suppress the amplitude fluctuations inherent at the lower frequencies [27].

Another interesting feature of diode lasers is that sidebands can be generated even at frequencies as high as a few $\mathrm{GHz}$ by fast modulation of the injection current. Heterodyne detection methods can then be easily implemented.

Extended cavity diode lasers can be easily locked on absorption lines, which can also be of interest for this experiment. In fact, the centre frequency must be controlled even for the long recording time required for signal integration. This can be accomplished either using an accurate $\lambda$-meter or by looking at the beat note of the probing laser with a second laser locked on a nearby frequency reference $\left(a^{16} \mathrm{O}{ }^{18} \mathrm{O}\right.$ line, for example).

b) Absorption cell: in order to achieve maximum sensitivity, a cell as long as possible should be used. Using multipass cells it is easy to achieve $100-200 \mathrm{~m}$ path length.

As already mentioned above, when transitions starting from the lowest rotational levels are observed, a way to increase the detection sensitivity is to reduce the temperature in the cell. A factor of 10 increase in sensitivity for the relevant transitions can be achieved in this way.

c) Detection: as already mentioned, an important feature of semiconductor diode lasers is their low-intensity noise which can be close to the shot-noise limit. This allows to achieve a very high sensitivity even by simple absorption detection. However, in order to reduce the effect of low-frequency noise, frequency modulation techniques can be used. The simplest method is to modulate the laser frequency with a relatively slow modulation $(\sim 50 \mathrm{kHz})$ and use lock-in detection.

For maximum sensitivity, a high-frequency $(\sim \mathrm{GHz})$ heterodyne detection method is possible. Different methods have been demonstrated [28-30]. A minimum absorption as low as $10^{-7}$ in a $1 \mathrm{~Hz}$ bandwidth can be detected in this way[31].

43. Expected accuracy. - The purpose of the proposed experiment is to measure the ratio of the intensities of the lines starting from antisymmetric and symmetric 
states. As discussed above, this corresponds to measuring the ratio of the populations of the two states, that is $\beta^{2}$.

The accuracy one can expect to achieve in the determination of the value, or an upper limit, for $\beta^{2}$ can be calculated using for the relevant experimental parameters the values given in subsect. $4 \cdot 2$, as follows.

The minimum absorption one can detect using high-sensitivity detection schemes is of the order of $10^{-7}$ in a $1 \mathrm{~Hz}$ bandwidth.

The absorption coefficient of the observed $\mathrm{O}_{2}$ lines starting from rotational levels close to the relevant ones, is $\sim 5 \cdot 10^{-7} \mathrm{Torr}^{-1} \mathrm{~cm}^{-1}$ at $300 \mathrm{~K}$. It can be increased by about one order of magnitude by reducing the temperature of the gas to $100 \mathrm{~K}$.

Therefore, using a multipass cell with a path length of $100 \mathrm{~m}$, a gas pressure of 100 Torr, and signal averaging for 400 seconds, it is possible to set a limit of $10^{-9}$ for $\beta^{2}$. This limit can in principle be further reduced to less than $10^{-10}$ using, for example, longer averaging times and a cell with a longer path length. In this case, however, other detection techniques, such as laser multistep photoionization, might be more appropriate.

The performances assumed in this calculation require a rather sophisticated but realistic apparatus. In fact, a comparison with the data in subsect. 3.2 shows that even with less demanding performances, such an experiment would allow an improvement in accuracy by several orders of magnitude with respect to existing data.

\section{5. - Conclusions.}

Although the possibility of a small violation of the Bose or Fermi statistics is still an open question from the theoretical point of view, the low accuracy of existing experimental tests for bosons makes a simple experiment like the one proposed here tempting and worth being tried. Using standard methods of high-sensitivity molecular spectroscopy, an upper limit of $10^{-9}-10^{-10}$ can be set on the probability of occupation of states violating the symmetrization postulate. This would represent an improvement of several orders of magnitude with respect to existing experimental data and would place stringent bounds to the validity of theories implying such violations.

I wish to thank $0 . \mathrm{W}$. Greenberg for sending me a preprint of a paper on quons and R. C. Hilborn for giving me information about his experiment mentioned in the text. I acknowledge useful discussions with V. I. Man'ko, G. Marmo, E. C. G. Sudarshan, and F. Zaccaria.

\section{REFERENCES}

[1] G. Gentile: Nuovo Cimento, 17, 493 (1940).

[2] H. S. Green: Phys. Rev., 90, 270 (1953).

[3] A. Yu. Ignat' ev and V. A. Kuz'min: Sov. J. Nucl. Phys., 46, 444 (1987).

[4] L. B. OKun: JETP Lett., 46, 529 (1987). 
[5] O. W. Greenberg and R. N. Mohapatra: Phys. Rev. Lett., 59, 2507 (1987); Erratum, 61, 1432 (1988); Phys. Rev. Lett., 62, 712 (1989).

[6] R. N. Mohapatra: Phys. Lett. B, 242, 407 (1990).

[7] D. I. Fivel: Phys. Rev. Lett., 65, 3361 (1990); Erratum, 69, 2020 (1992).

[8] O. W. Greenberg: Phys. Rev. D, 43, 4111 (1991).

[9] D. I. FIVEL: Phys. Rev. A, 43, 4913 (1991).

[10] L. C. Biedenharn: J. Phys. A, 22, L873 (1989).

[11] F. WILCZEK: Fractional Statistics and Anyon Superconductivity (World Scientific, Singapore, 1990).

[12] V. I. Man'ko, G. Marmo, S. Solimeno and F. Zaccaria: Int. J. Mod. Phys. A, 8, 3577 (1993).

[13] V. I. Man'ko, G. Marmo, S. Solimeno and F. Zaccaria: Phys. Lett. A, 176, 173 (1993).

[14] E. Ramberg and G. A. Snow: Phys. Lett. B, 238, 438 (1990).

[15] See, for example, G. Herzberg: Spectra of Diatomic Molecules, 2nd edition (D. Van Nostrand Co., Princeton, N.J., 1950).

[16] 0. W. Greenberg: Physica A, 180, 419 (1992).

[17] A. B. Govorkov: Physica A, 203, 655 (1994).

[18] T. Curtright, D. Fairlie and C. Zachos (Editors): Proceedings of the Workshop on Quantum Groups (World Scientific, 1991).

[19] R. P. Feynman, R. B. Leighton and M. SAnds: The Feynman Lectures on Physics, Vol. III, p. 4.

[20] O. W. Greenberg and R. N. Mohapatra: Phys. Rev. D, 39, 2032 (1989) and references therein.

[21] D. E. Kelleher, J. D. Gillaspy and K. Deilamian: in Laser Spectroscopy IX, edited by M. S. Feld, J. E. Thomas and A. Mooradian (Academic Press, 1989) p. 332.

[22] R. Hilborn: Bull. Am. Phys. Soc., 35, 982 (1990). In this experiment, based on an idea similar to the one discussed in this paper, the dipole-allowed (001)-(000) vibrational transitions in $\mathrm{C}^{16} \mathrm{O}_{2}$ would be investigated.

[23] See, for example, Applied Laser Spectroscopy, edited by W. DemTroder and M. INGUSCIO, NATO ASI Series (Plenum, New York, N.Y., 1990).

[24] M. DE Angelis, F. Marin, F. Pavone, G. M. Tino and M. Inguscio: in Monitoring of Gaseous Pollutants by Tunable Diode Lasers, edited by R. GRISAR, H. BotTNER, M. TACKE and G. Restelli (Kluwer Academic Publishers, Dordrecht, 1992); M. DE ANGelis, G. Pesce, A. Sasso, G. M. Tino and F. PAvone: IV European Quantum Electronic Conference EQEC'93, Firenze, 1993.

[25] C. E. Wieman and L. HollberG: Rev. Sci. Instrum., 62, 1 (1991).

[26] G. M. Tino: Phys. Scr., T51, 58 (1994).

[27] L. Hollberg, R. Fox, N. Mackie, A. S. Zibrov, V. L. Velichansky, R. Ellingsen and H. G. Robinson: in $X$ International Conference on Laser Spectroscopy, edited by M. Ducloy, E. Giacobino and G. CAMy (World Scientific, 1992), p. 347.

[28] G. C. BJoRKLUND: Opt. Lett., 5, 15 (1980).

[29] G. R. Janik, C. B. Carlisle and T. F. Gallagher: J. Opt. Soc. Am. B, 3, 1070 (1986).

[30] C. B. Carlisle and D. E. CoOPer: Opt. Lett., 14, 1306 (1989).

[31] F. S. Pavone and M. Inguscio: Appl. Phys. B, 56, 118 (1993).

Questo periodico all'Unione Stampa Periodica Italiana 